\title{
Infrared imaging of TiNi shape memory alloy subjected to deformation at various temperatures
}

\author{
E.A. Pieczyska ${ }^{1, a}$ and H. Tobushi ${ }^{2}$ \\ ${ }^{1}$ Institute of Fundamental Technological Research, PAS, Pawinskiego 5B, 02-106 Warsaw, Poland \\ ${ }^{2}$ Department of Mechanical Engineering, Aichi Institute of Technology, 1247, Yachigusa, Yakusa- \\ cho, Toyota-city, AICHI, 470-0392, Japan
}

\begin{abstract}
Summary. The infrared imaging of stress-induced martensitic transformation occurring in TiNi shape memory alloy subjected to deformation in various conditions has been presented. During the loading and unloading processes the mechanical parameters and the infrared radiation from the specimen surface have been continuously recorded by infrared camera. Basing on the obtained stress-strain curves and the elaborated temperature changes it was found that onset of the martensitic transformation appears at the end of the elastic part of the stress-strain curve, since a temperature increase was noticed there. The uniform temperature distribution observed there means that the initial transformation started at this stage is macroscopically homogeneous. It is followed by the martensitic intense transformation, related to the significant temperature increase. The more advanced transformation, the higher temperature changes. For the shape memory alloy pseudoelastic behavior the narrow bands of higher temperature are observed, manifesting localized Lüders-like deformation. For the shape memory effect behavior the process of transformation is much more homogeneous and characterized by uniform temperature distribution. Using a modern, sensitive and fast dynamic infrared camera enables to obtain new results of the start and development of the martensite forward and reverse transformation.
\end{abstract}

\section{Introduction to shape memory alloys}

The development of intelligent or smart materials and their systems is highly expected since they have various functions, such as sensing, working and crack-healing by themselves, etc. In the intelligent materials, the development of shape memory alloy (SMA) has attracted high attention since the unique properties of the shape memory effect (SME) and superelasticity (SE) appear. If we use the SME and SE in practical applications, not only large recovery strain but also high recovery stress, energy storage and energy dissipation can be obtained. Successful applications of shape memory effect are couplings, actuators in electric appliances, automobile devices, robotics and smart materials, e.g. composites with polymer or metal matrix. Pseudoelasticity and high elasticity are applied to orthodontic arch wire, antennas for cellular phones, guide wires for catheters, medical instruments, orthopedic devices, etc. [1-11].

\footnotetext{
a e-mail : epiecz@ippt.gov.pl
} 
The shape memory alloy deformation behavior, related to many practical applications, is summarized graphically by the three-dimensional stress-strain-temperature diagram in Figure 1 [2].

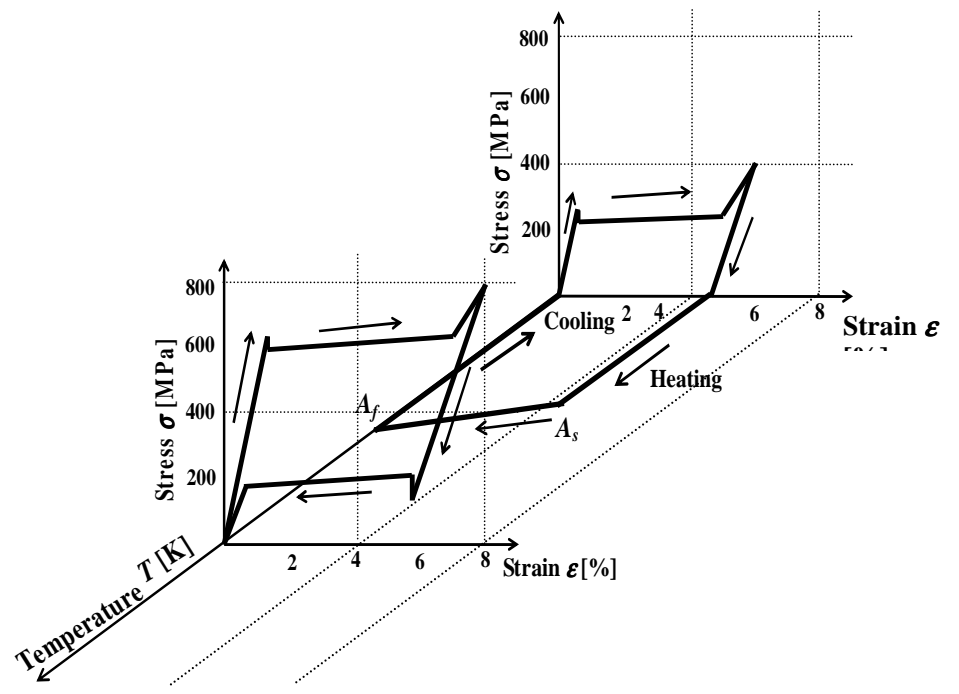

Fig. 1. Three-dimensional stress-strain-temperature diagram showing the deformation and shape memory and superelastic behaviors of a Ti-Ni alloy deformed below $A_{s}$ and above $A_{f}$.

In the Figure, $A_{s}$ and $A_{f}$ denote the austenite start and finish temperatures, respectively. In the yield stage of the stress-strain diagram shown in the $\sigma-\varepsilon$ plane, the deformation accompanied with stress plateau appears due to the martensitic transformation. This phenomenon is called the stress-induced martensitic transformation (SIMT). The strain induced at a temperature below $A_{s}$, 6\%, recovers between $A_{s}$ and $A_{f}$ after the applied stress has been removed and the specimen heated, as seen in the $\varepsilon-T$ plane, showing the shape memory effect (SME). At a temperature above $A_{f}$ the SIMT is formed, leading to the usual superelastic (SE) loop with upper and lower plateaus.

The main features of the SME and SE are induced due to the martensitic transformation. Since the shape memory alloys are very sensitive to the temperature changes, effects of thermomechanical couplings related to the stress-induced martensitic transformation responsible for their behavior and related to many effective applications can be studied on the basis of the obtained mechanical and temperature characteristics. To this end a particular program for TiNi shape memory alloy thermomechanical investigation is proposed.

\section{Experimental techniques}

The experiments have been carried out on two kinds of TiNi shape memory alloy belt type specimens of $160 \times 10 \times 0.4 \mathrm{~mm}$, cut off from strips; characterized by the $A_{f}$ temperature equal to $333 \mathrm{~K}$ and $283 \mathrm{~K}$, respectively. The specimens have been subjected to the strain-controlled tension test on Instron testing machine in quasi-static range of the strain rates. In the course of investigation both the mechanical characteristics and the distribution of the infrared radiation emitted by the specimen surface were registered continuously. Schematic and photographs of the experimental setup is presented in Figure 2. The used infrared camera is a long wave type, working in the wave range of $7.5-13 \mu \mathrm{m}$. The matrices size is $320 \times 240$ pixels. The spatial and the temperature resolutions depend on the camera-specimen distance. In the case of the results presented here, the distance was $10 \mathrm{~cm}$ and the spatial resolution was $0.3 \mathrm{~mm}$. Before the testing, the specimen surface had been 
covered with a very thin layer of a carbon black powder in order to make its emissivity higher and more homogeneous. The measurement temperature sensitivity was below $0.08 \mathrm{~K}$.

The temperature distribution on the specimen surface reflects immediately the onset and the development of the new phases, both martensite and reverse, due to the temperature changes and significant temperature variations between the parent and the new phase.

The average temperature was calculated over an area of $8 \mathrm{~mm} \times 60 \mathrm{~mm}$, located in the central part of the specimen. The average temperature was usually used in the presented curves and thermomechanical coupling analysis $[3,4,6]$.
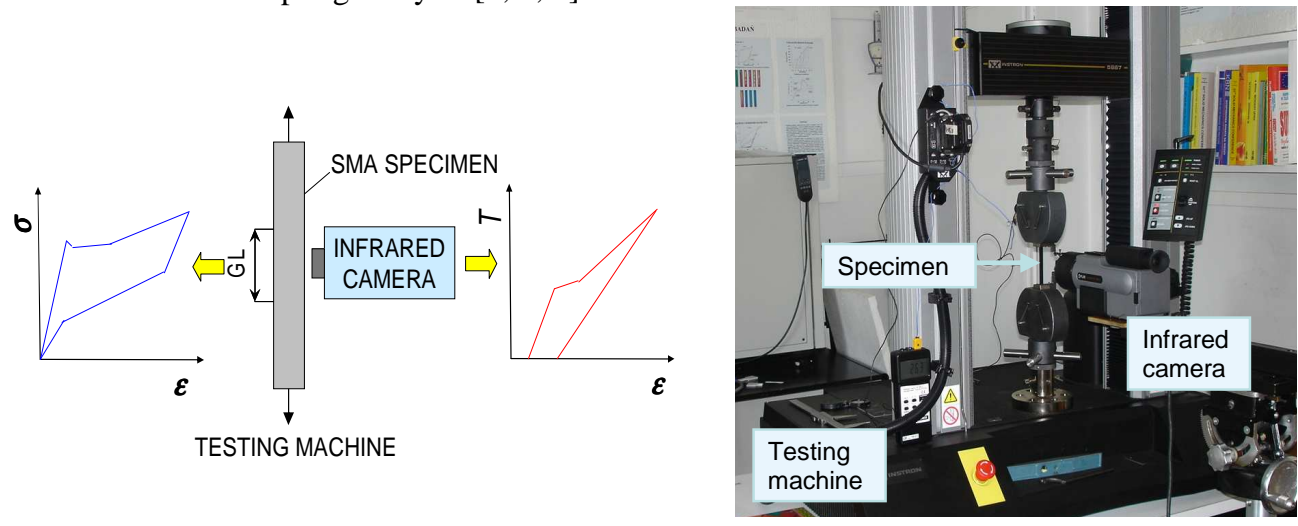

Fig. 2. Schematic and photograph of experimental set-up for mechanical and temperature investigation.

The obtained temperature and mechanical data enable study of the process of nucleation and further development of the martensitic forward and reverse transformation, responsible for the shape memory alloy thermomechanical behavior.

\section{Infrared imaging of stress-induced martensitic transformation}

The stress-induced martensitic transformation manifests an exothermic character, while the reverse endothermic one, so during loading the SMA temperature increases and during unloading the temperature decreases, respectively. The temperature changes depend on the strain rate; the higher the strain rate, the higher the temperature change [1-6]. The recorded maximal temperature increments were of about $4 \mathrm{~K}$ for the strain rate $10^{-4} \mathrm{~s}^{-1}$ to about $40 \mathrm{~K}$ for $10^{-1} \mathrm{~s}^{-1}$ [6]. Depending on the test temperature in reference to the SMA austenite finish $\left(A_{f}\right)$ and austenite start $\left(A_{s}\right)$ temperature parameters, the recorded temperature distribution was more or less uniform. It means that the process of the martensite transformation, analyzed in macro-scale, was more or less homogeneous. Investigations into measurements of the temperature variations accompanying the Martensitic transformation processes in shape memory alloys have been launched quite recently. At first, the temperature was estimated using a thermocouple, which, however, limited the measurement to a single chosen point only. Application of the infrared camera allows monitoring of the temperature distribution on the examined specimen surface, measurement of the mean temperature in contact-less way over arbitrary chosen area or points [3].

\subsection{Infrared imaging of shape memory effect}

Figure 3 presents the stress-strain curve and the specimen temperature distributions obtained during tension of the TiNi SMA $\left(A_{s}=320 \mathrm{~K}, A_{f}=333 \mathrm{~K}\right)$ at $295 \mathrm{~K}$, below the SMA austenite start temperature, i.e. registering shape memory effect [5]. The images presented in left show the specimen temperature distribution recorded at strains equal to $0,1 \%, 2 \%, 3 \%$ during loading; and $2.9 \%$ during unloading. 

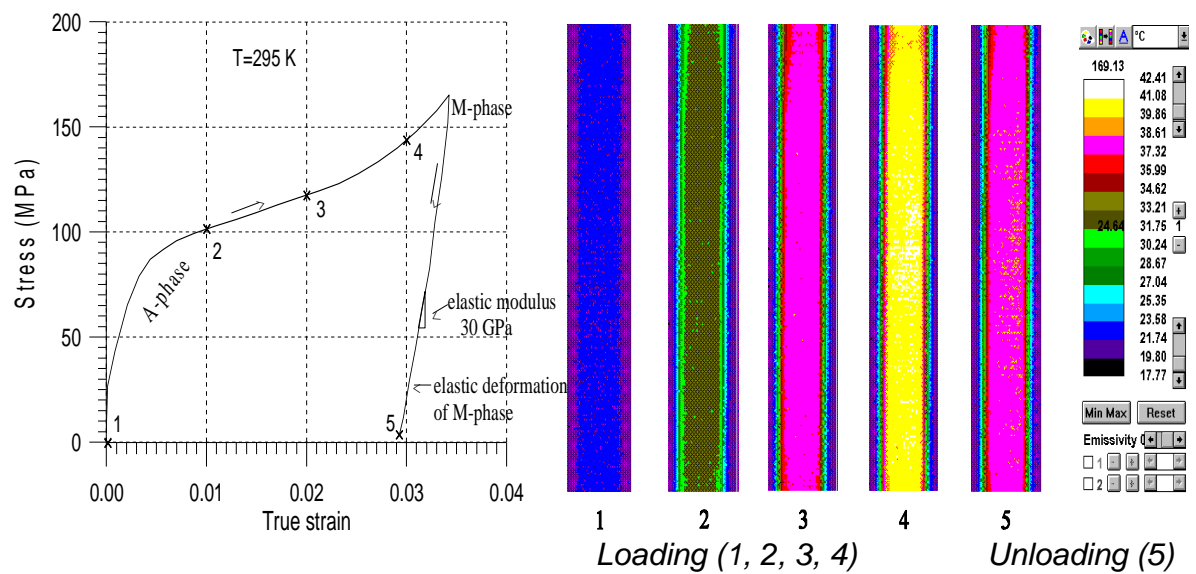

Fig. 3. Stress-strain curve and temperature distribution on the surface of TiNi SMA obtained at various strains during loading and unloading at $\mathrm{T}<\mathrm{A}_{\mathrm{s}}$.

One can find from the figure that the temperature increases during the SMA loading and decreases during the unloading but the temperature distribution was uniform, i.e. the martensitic transformation process is homogeneous. After unloading, a great residual strain $\approx 3 \%$ called the shape memory effect was recorded. The specimen recovers its former size and shape during subsequent heating at the temperature between $320 \mathrm{~K}$ and $333 \mathrm{~K}$.

\subsection{Infrared imaging of superelasticity}

For the TiNi SMA characterized by the $A_{f}=283 \mathrm{~K}$ and subjected to tension test carried out at room temperature $(295 \mathrm{~K})$ the temperature imaging is much more complex (Figure 4 ), reflecting the much more complicated transformation process [6].

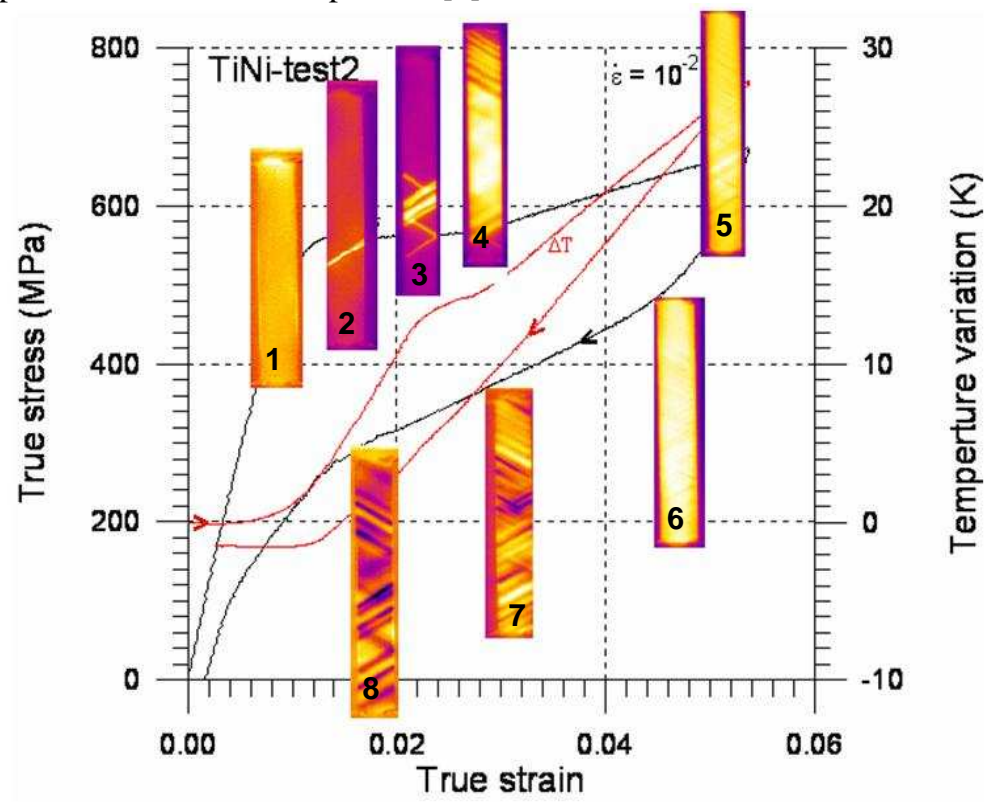

Fig. 4. Stress- and temperature-strain curves completed by the infrared temperature distributions obtained for TiNi SMA tension test performed with strain rate $10^{-2} \mathrm{~s}^{-1}[6]$. 
Looking at Figure 4 one can observe bands of significantly higher temperature, corresponding to the exothermic inhomogeneous Martensitic forward transformation recorded during the SMA loading $(1-5)$, whereas bands of significantly lower temperature, corresponding to the endothermic reverse transformation, were recorded during unloading (6-8). At higher strains, more and more lines evolving into the bands appear and move towards the specimen grip. When the bands reach the grips region (4), the upswing region on both the stress-strain and the temperature curves is observed, reflecting more advanced stage of the transformation process. The stress and the temperature increase fast, but the temperature distribution becomes more uniform (5), so the transformation process is more homogeneous at this advanced transformation stage. During the unloading, after passing its elastic stage (6), the reverse transition initiates. After the unloading is completed, the material almost returns to its parent austenite phase. However, the temperature drops below the initial one and the residual strains, related to a small amount of martensitic and irreversible macrostructural changes appear, depending on the specimen history [6, 7].

The phenomena of localized Lüders-like transformation accompanying the complete loop of the TiNi SMA pseudoelasticity behavior analyzed in infrared technique $[1-6,8,11]$ have been confirmed by Daly et al. by using a Digital Image Correlation (DIC) Strain Map technique (Figure 5), [7].

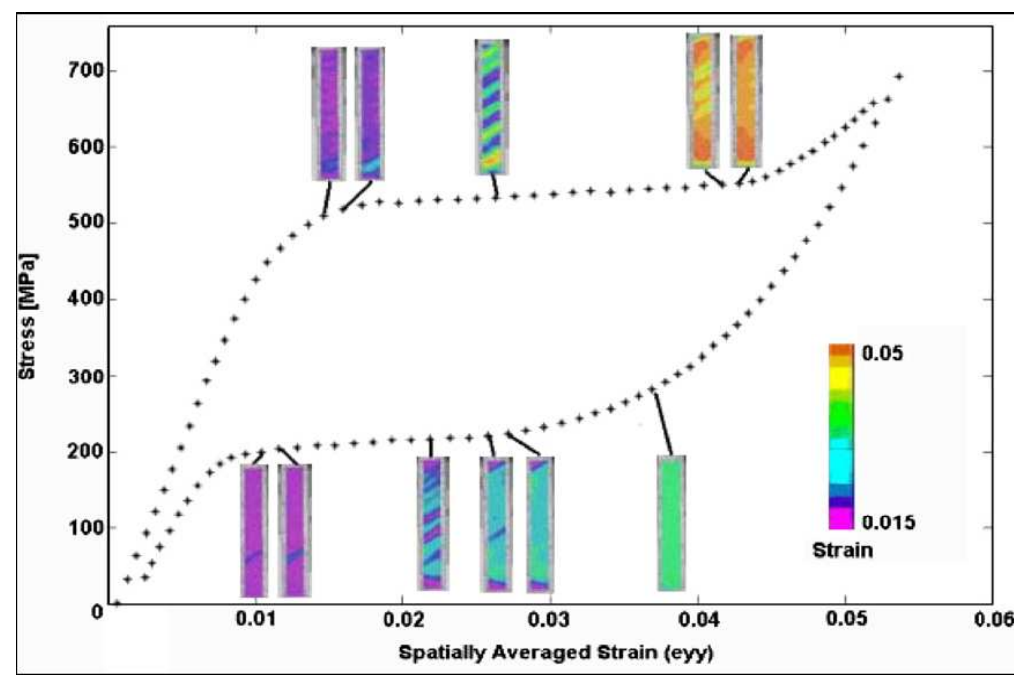

Fig. 5. The macroscopic response of TiNi SMA stress-strain curve with corresponding DIC images; S. Daly, G. Ravichandran, K. Bhattacharya, Acta Materialia 55 (2007) [7].

The macroscopic response presented in Figure 5 is the spatially averaged strain value from each DIC image plotted vs. the MTS stress value. The not uniform strain distribution obtained on the specimen surface reflects inhomogeneous and evaluating process of the martensitic transformation.

\subsection{Infrared imaging of the transformation onset}

Various techniques have confirmed the localized martensitic transformation related to the SMA pseudoelastic behaviour, whereas it is still difficult to clear up the onset of the transformation. Therefore, particular attention was paid in our approach to the beginning of the process. Application of high resolution infrared camera enables us to demonstrate even some symptoms, preceding the phase transformation (Figure 6; b, c). Namely, after the piezocalorimetric effect, i.e. fine temperature decrease recorded by the IR system $(0.01 \mathrm{~K})$, a small but systematically increasing uniform temperature change was recorded even on elastic part of the stress-strain curve, manifesting initial homogeneous stage of the transformation process (Figure 6; b). Then, around the "Yield point", narrow bands of significantly higher temperature from the other part of the specimen, were recorded 


\section{EPJ Web of Conferences}

manifesting localized, martensitic forward transformation (Figure 6; $-\mathrm{h}$ ). It was proved in the tests and published in former papers that the first indication of localized martensitic transformation occurs for both the stress and strain-controlled tests at the strain value $\varepsilon$ of about 0.013 . At the beginning, it was just a line of significantly higher temperature started from the specimen left or right edge (probably surface defect), evolving into a narrow band (Figure $6 \mathrm{~d}$ ). The band made an angle of about $48^{\circ}$ with the direction of tension.

a)

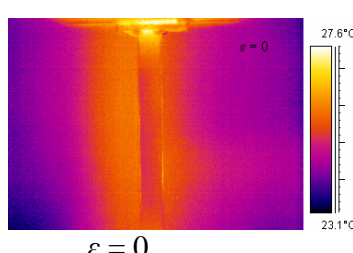

e)

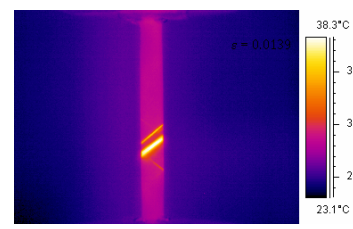

$\varepsilon=0.0139$ b)

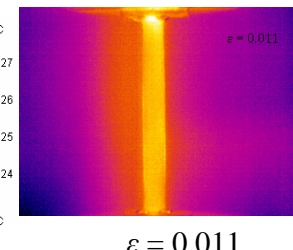

f)

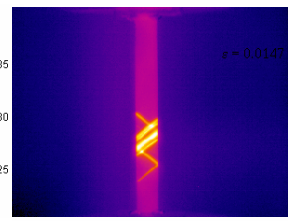

$\varepsilon=0.0147$ c)

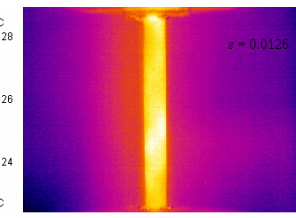

$\varepsilon=0.0126$

g)

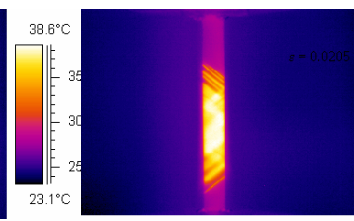

$\varepsilon=0.0205$ d)

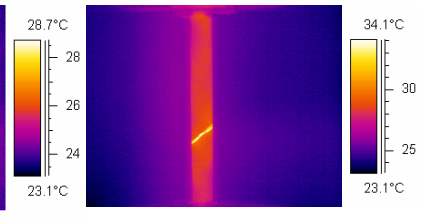

$\varepsilon=0.0130$

h)

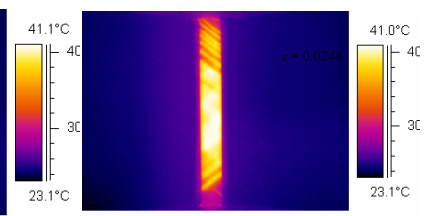

$\varepsilon=0.0244$

Fig. 6. Infrared temperature distributions obtained at initial stage of TiNi SMA tension test: $\varepsilon=0-0.0244$.

As the tension proceeds, the band widens gradually and other bands appear (Figure 6; e - h). At first, new parallel bands appear, and then the new bands develop at the same angle but in opposite direction $[3,4,6]$. The temperature difference in the area where the band appears is about $8 \mathrm{~K}$, proving the rapid nature of the process. The highest temperature was registered at the intersection of the bands.

\section{New applications of shape memory alloys to technology and medicine}

Due to unique properties of shape memory alloys manifested in various conditions, many practical applications are still developing, both to medicine and technology.

\subsection{New artificial anal sphincter using a two-way shape memory alloy effect}

The authors created a new artificial anal sphincter using a shape memory alloy (AS-SMA) to treat fecal incontinence and evaluated its validity [9]. It consists of two Ti-Ni plates to sandwich the intestine and generates a pressure of $55 \mathrm{~mm} \mathrm{Hg}$ at its resting position. With the electric power supply, the two metals bend to form an almond-like shape making a maximum gap of $33 \mathrm{~mm}$ between each other at the temperature of $55^{\circ} \mathrm{C}$. A transcutaneous energy transmission system was used to supply power to the artificial sphincter. The system consisted of two coils, one outside and the other inside the body. An overheating protector, including silicone coating was used to secure the tissue. The complete device was attached to the colostomy in a piglet and was operated several times a day for one month. Fecal continence, bowel movements, and general condition of the piglet were recorded. At the autopsy, no compression injury was detected. With reduction of the associated side effects, the completely implantable AS-SMA device may achieve fecal continence with fewer complications than other surgical treatments and could be a potential substitute for an impaired anal sphincter. 

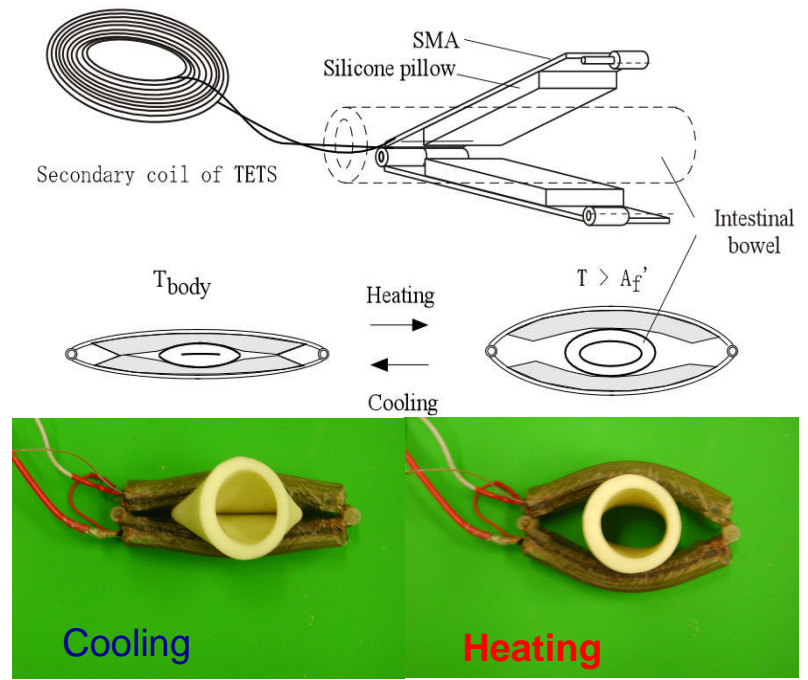

Fig. 7. Project of new artificial anal sphincter using a two-way shape memory alloy effect K. Nishi, T. Kamiyama, M. Wada, S. Amae, T. Ishii, T. Takagi, Y. Luo, T. Okuyama, T. Yambe, Y. Hayashi, R. Ohi, J. Pediat. Surg., 39, 1 (2004) [9].

\subsection{Solar vehicle based on shape memory effect in TiNi SMA}

A photograph of a solar-powered car driven a simple-pulley SMA heat engine is shown in Figure 8 [10].

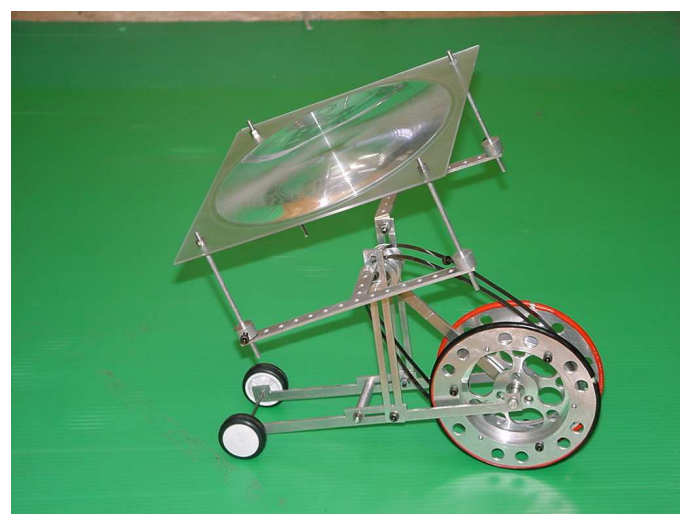

Fig. 8. Project of new solar vehicle using a shape memory alloy effect,

H. Tobushi, K. Date, K. Miyamoto, Proc. APCMM (2009) [10].

The SMA heat engine is driven by the sun light using a Fresnel lens. In the SMA heat engine, two pulleys are connected by the SMA belt which is shape-memorized in the flat plane. The car moves based on the recovery force in the SMA belt which appears by heating from the sun light focused by the Fresnel lens. In order to use the low-temperature thermal energy around $373 \mathrm{~K}$ from a view-point of global warming prevention, the development of the compact and harmless clean SMA heat engine is greatly expected.

\subsection{Opening and closing door driven by SMA thin strips}

Photographs of the rotary movement of an opening and closing door model using two kinds of shape memory alloy thin strips are shown in Figure 9. SME-SMA strip shows the shape memory effect 


\section{EPJ Web of Conferences}

(SME) whereas the SE-SMA strip shows SE (superelasticity) at room temperature under heating and cooling. The SME-SMA strip was heated by Joule heat effect through electrical current [11].

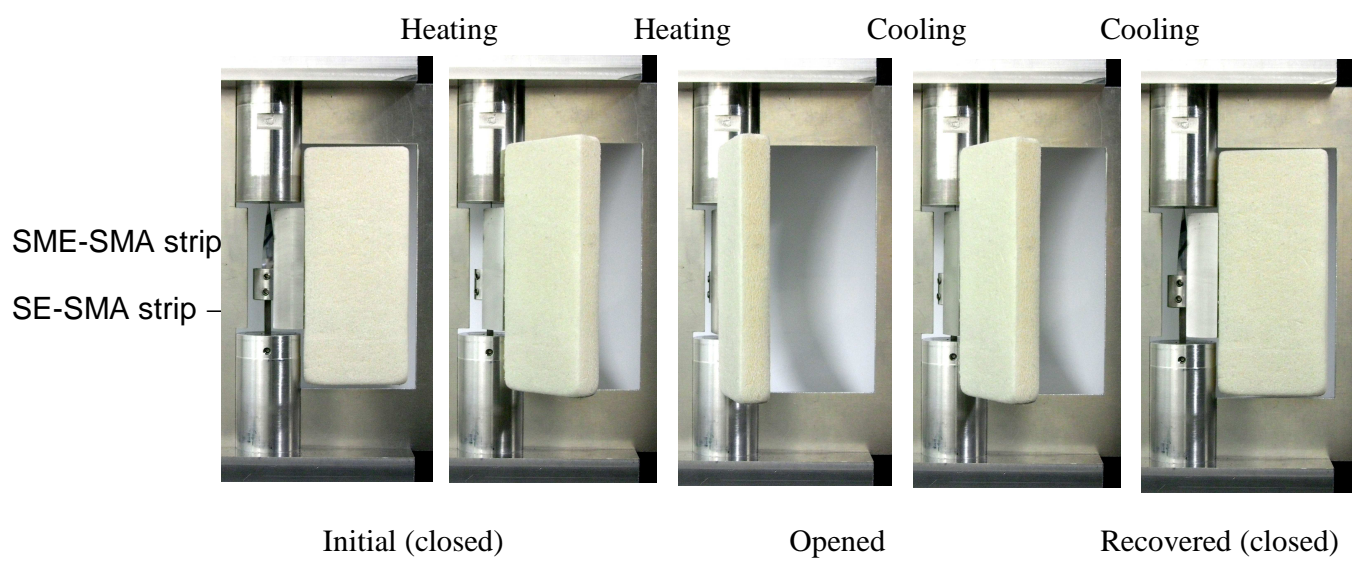

Fig. 9. Photographs of two-way rotary movement of a door driven by SMA thin strips during heating and cooling, H. Tobushi, E.A. Pieczyska, W.K. Nowacki and Y. Sugimoto, Solid State Phenom., 154, (2009) [11].

As can be seen in the figure, the door is closed in the initial state, since torque of SE-SMA strip $M_{S E}$ is larger than that of the SME-SMA strip $M_{S M E}$. Since recovery torque appears by heating the SME-SMA strip and the relation of the torque changes into $M_{S M E}>M_{S E}$, the SME-SMA strip recovers a flat plane and therefore the door is opened. When the SME-SMA strip is cooled thereafter, the relation of torque varies again into $M_{S M E}<M_{S E}$. Therefore, the SE-SMA strip recovers a flat plane, resulting in closing the door. Thus, if two kinds of SMA thin strips which show the SME and SE are used, a two-way rotary driving element with a small and simple mechanism can be developed and applied, for example, to control the door position.

\section{Acknowledgments}

The research has been carried out with partial support of the Japan Society for the Promotion of Science; Post-doc ID P04774, and by the Polish Ministry of Science and Higher Education; Grant No 501220837. The support of Prof. W. K. Nowacki in performing this research work and the assistance of Dr. S. P. Gadaj in obtaining the experimental data are appreciated.

\section{References}

1. J.A. Shaw and S. Kyriakides, Acta Mater., 45, 2, (1997)

2. E.A. Pieczyska, Y. Ejiri and T. Sakuragi, Mech. Adv. Mater. Struct., 16 (2009)

3. E.A. Pieczyska, S.P. Gadaj, W.K. Nowacki and H. Tobushi, Exp. Mechanics, 46, 4 (2006)

4. E.A. Pieczyska, S.P. Gadaj, W.K. Nowacki and H. Tobushi, Bull. Pol. Ac., Tech. 52-3 (2004)

5. S.P. Gadaj, W.K. Nowacki and E.A. Pieczyska, Infrared Physics \& Tech., 43 (2002)

6. Pieczyska E., Prace IPPT/ IFTR Reports, ISBN 978-83-89687-37-1, 3, (in Polish, graphs in English) (2008)

7. S. Daly, G. Ravichandran and K. Bhattacharya, Acta Mater., 55, (2007)E. Pieczyska, W. Nowacki, T. Sakuragi and H. Tobushi, Key Eng. Materials, 340-341 (2007)

9. K. Nishi, T. Kamiyama, M. Wada, S. Amae, T. Ishii, T. Takagi, Y. Luo, T. Okuyama, T. Yambe, Y. Hayashi and R. Ohi, J. Pediatr. Surg., 39, No 1 (2004)

10. H. Tobushi, K. Date and K. Miyamoto, Proc. APCMM (2009)

11. H. Tobushi, E.A. Pieczyska, W.K. Nowacki and Y. Sugimoto, Solid State Phenom., 154, (2009). 